\title{
Immediate Removal of an Entrapped Central Venous Catheter Guide Wire
}

\author{
Jan Bruthans ${ }^{1,2}$, Stanislav Trča ${ }^{3}$ \\ ${ }^{1}$ Department of Anesthesiology and Intensive Care, First Faculty of Medicine, \\ Charles University and General University Hospital in Prague, Prague, Czech \\ Republic; \\ 2Department of Biomedical Technology, Faculty of Biomedical Engineering, \\ Czech Technical University in Prague, Prague, Czech Republic; \\ ${ }^{3}$ First Department of Surgery - Department of Abdominal, Thoracic Surgery \\ and Traumatology, First Faculty of Medicine, Charles University and General \\ University Hospital in Prague, Prague, Czech Republic
}

Received November 30, 2018; Accepted May 2, 2019.

Key words: Central venous line - Guide wire entrapment - Venous angle Thoracic surgery

\begin{abstract}
Extravasal guide wire entrapment due to fraying during central venous catheter placement using the Seldinger technique is a rare complication, which should be resolved as soon as possible. A 68-year-old male was scheduled for open right-side decortication. After the induction of general anesthesia, an attempt was made to place a central venous line in the right subclavian vein. However, the guide wire was entrapped extravasally between the right clavicle and the first rib. The exact site was located by palpating the bend of the guide wire in the subclavian triangle and the thoracic surgeon was available. Therefore, it was decided not to try to visualize the guide wire any further and to immediately proceed with surgical removal of the guide wire. The platysma muscle was dissected allowing access to the subclavian triangle. Venotomy of the external jugular vein was performed and the entrapped guide wire was removed via the venotomy. The whole complication was resolved within 30 minutes and the primary procedure was then performed. Managing rare complications of central venous line placement requires skill, ingenuity and, sometimes, interdisciplinary cooperation, either with a radiologist or a surgeon. The decision to proceed with immediate surgical removal of the guide wire proved a right one, and, to the best of our knowledge, such a strategy has not been described in the relevant literature to date.
\end{abstract}

Mailing Address: Jan Bruthans, MD., PhD., Department of Anesthesiology and Intensive Care, First Faculty of Medicine, Charles University and General University Hospital in Prague, U Nemocnice 2, 12808 Prague 2, Czech Republic; Phone: +420 723426 288; e-mail: jan@bruthans.cz 


\section{Introduction}

Central venous catheter placement is a widely used procedure performed, always using the Seldinger technique, at the $1^{\text {st }}$ Department of Surgery of General University Hospital in Prague in more than 500 cases each year. Also, typical complications (e.g. arrhythmias, arterial puncture, pneumothorax, hemothorax, air embolism, etc.) are commonly known and routinely dealt with. Much rarer ones include those involving guide wire insertion or removal, although even these have been described in the relevant literature - kinking (Katiyar and Jain, 2010) or knotting (Leigh et al., 1987), loss (Schummer, 2002) or fragmentation (Polos and Sahn, 1991) of the guide wire, or separation of the spiral part of the guide wire (Önal et al., 2013). However, most of the complications are not dealt with until after they have occurred, usually identified by $\mathrm{X}$-ray and subsequently managed surgically (Pal et al., 2014) or by interventional radiology (Khan et al., 2007). We would therefore like to present our case of central venous catheter guide wire entrapment due to fraying successfully resolved by open surgery within a mere 30 minutes of the incident.

\section{Case report}

A 68-year-old patient with a history of hypertension and ulcer disease had been repeatedly treated for two months prior to the event in General University Hospital in Prague, first due to a traffic accident (multiple left rib fractures, lung contusion, and hemothorax requiring thoracotomy) and, later, for colonic torsion (requiring left hemicolectomy). His postoperative state was complicated by confusion and later by right-sided pleural effusion managed twice by fluid aspiration (with 80 and $250 \mathrm{ml}$ of transudate removed). Multiloculated pleural effusion was diagnosed by CT (computed tomography) and the patient was scheduled for open right-side decortication.

The general anesthesia was induced by propofol, sufentanyl and rocuronium and the patient was intubated. Given the anticipated extent of the procedure and postoperative care, we decided to place a central venous line in the right subclavian vein using the lateral infraclavicular approach. We identified the best puncture site using standard body landmarks (clavicle and surrounding muscle tissue). Standard disinfection was performed, and barrier protection ensured.

The actual procedure was performed using $7 \mathrm{Fr}$ triple-lumen $20-\mathrm{cm}$ Arrow $^{(T M)}$ (Arrow International, Inc., Reading, USA) catheter. A third-year trainee anesthesiologist located the subclavian vein on the first attempt without complications. The Seldinger wire was passed through the needle, with just very slight resistance felt. The needle was subsequently removed, and a dilator introduced over the wire. Once the dilator had been removed, the catheter was threaded over the wire. While the catheter tip had been advanced beyond skin surface, the wire could not be withdrawn, and the guide wire seemed to have been entrapped. The catheter was eventually removed and a consultant anesthesiologist was called in. 


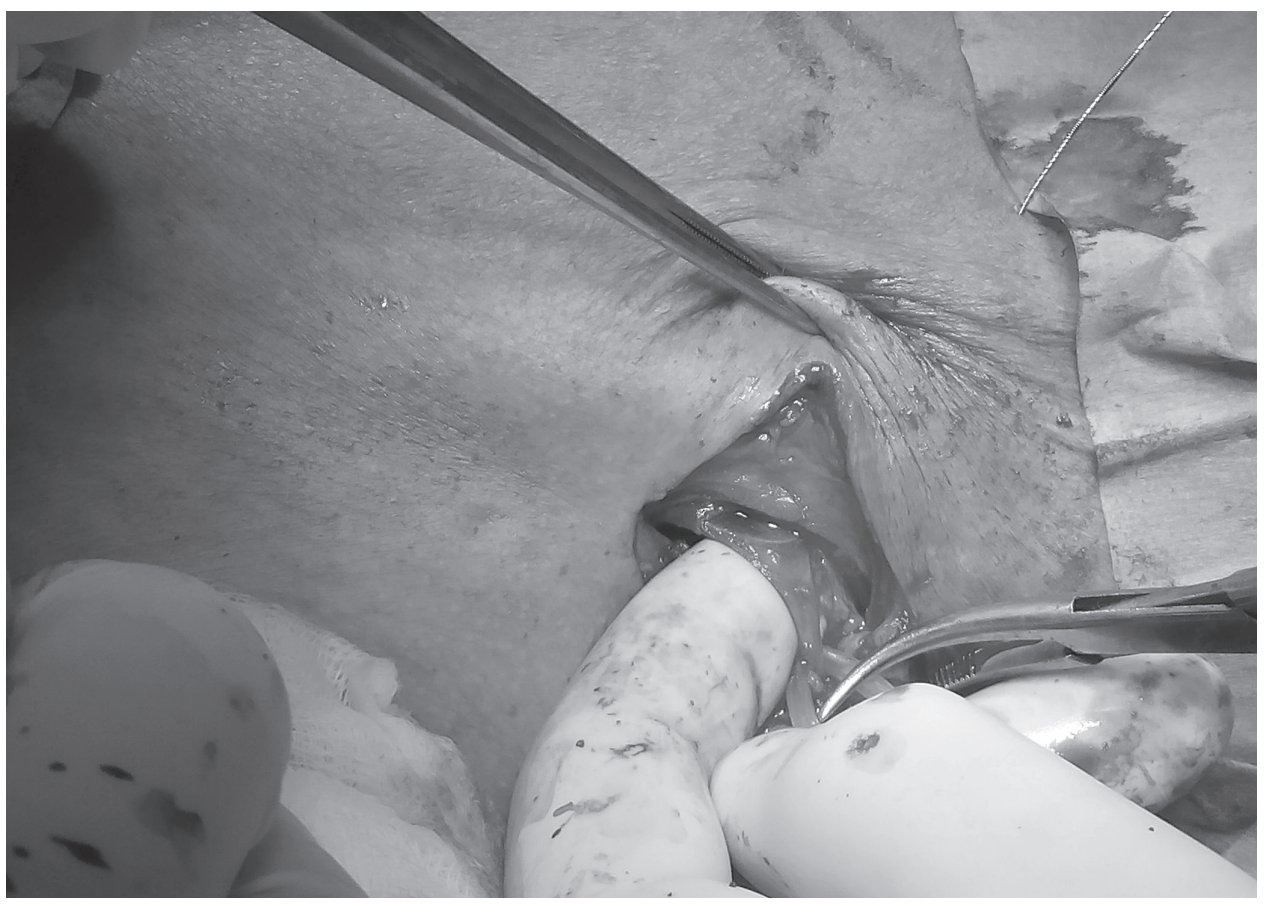

Figure 1 - The external jugular vein was freed, and a plastic tube used as a tourniquet.

First, gentle traction was exerted in an effort to extract the wire while alternately applying gentle pressure to insert it, but to no avail; the guide wire simply would not move in either direction.

Manoeuvring with the patient's shoulder and arm also failed to unblock the guide wire. When, subsequently, more force was applied, it seemed the guide wire would be easily extracted. However, after a few centimetres, the guide wire failed to move anymore and, when carefully inspected, its body seemed to be elongated and the spiral part partially separated from the inner one. By palpation in the supraclavicular area, in the subclavian triangle, it was possible to feel a cranially oriented concave loop or bend of the wire at a site corresponding anatomically to the venous angle. A movement of the soft tissue at the guide wire introduction site below the clavicle, slightly lateral to the medioclavicular line, was evident when traction to the wire was applied. The patient's history did not suggest any known anatomic variations of the respective part of the venous system or the upper thoracic outlet that could be possibly attributed to the guide wire entrapment. There were no changes on the ECG (electrocardiogram).

At that point, the consultant anesthesiologist with the head of the thoracic surgical team decided to carry out prompt open revision at the site of the palpable guide wire loop. 


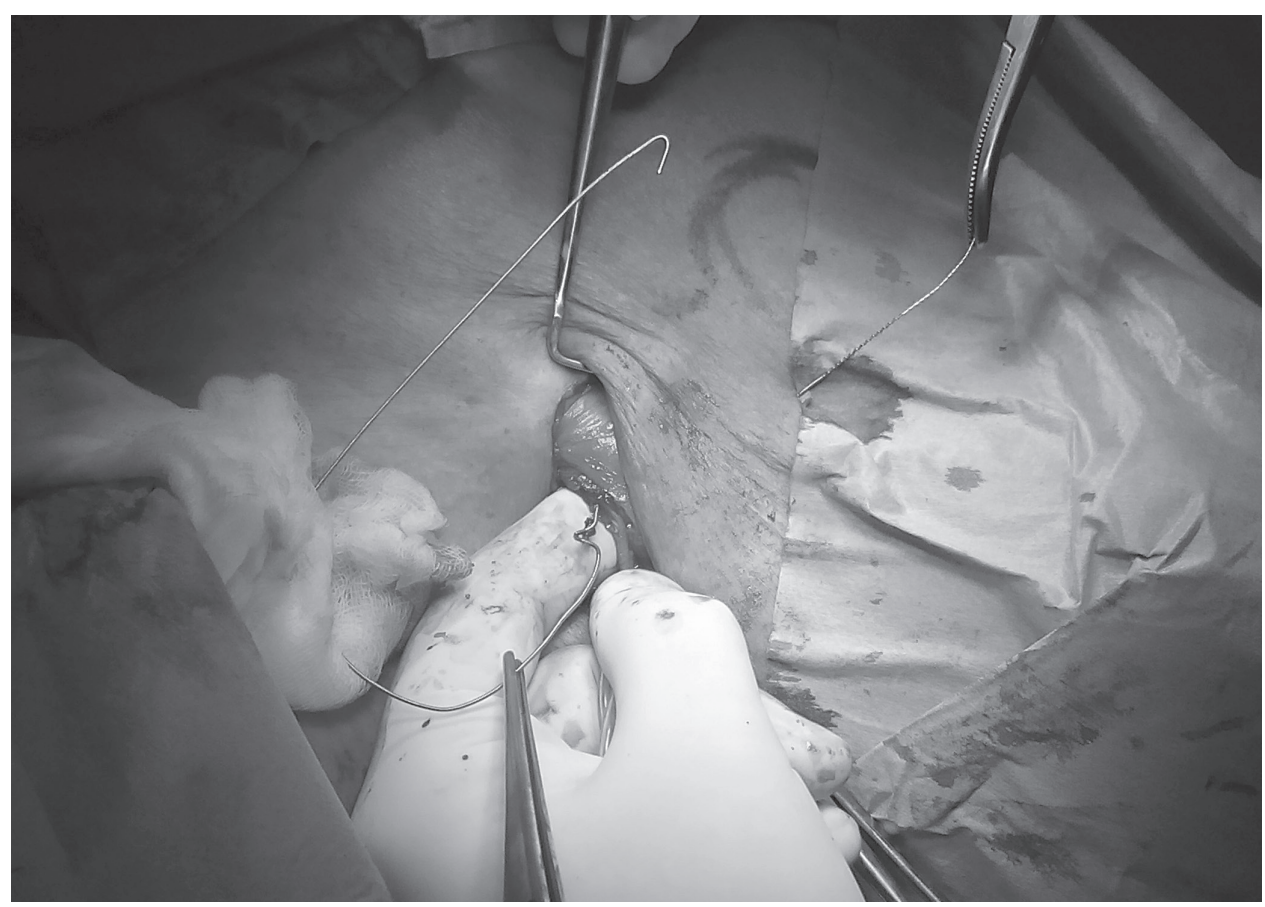

Figure 2 - The kinking of the guide wire was clearly visible.

Subsequently, standard disinfection was performed and barrier protection ensured. The part of the guide wire protruding from the skin was shortened. After skin incision, the platysma muscle was dissected allowing access to the subclavian triangle and reach the venous angle. At that point, an arc of the guide wire could be seen through the venous angle wall. The external jugular vein was freed, and a plastic tube used as a tourniquet (Figure 1). Venotomy of the external jugular vein was performed and the central (i.e. distal) part of the guide wire was smoothly withdrawn via the venotomy. The kinking of the guide wire was clearly visible (Figure 2). The guide wire was then clipped off just proximally to the kinking. Next, the remaining part of the wire (i.e. the medial part) was extracted via the same venotomy site, when the frayed area of the medial part of the guide wire was also clearly identified.

The guide wire (now in three parts) was inspected and found to have been completely removed. The venotomy site was closed; no vascular lesion occurred in the meantime. The wound was closed and the primary procedure (open right-side lung decortication) was then performed without further complications. It was decided not to place another central venous line, but to opt for two large-bore peripheral catheters. 
Upon recovery from anesthesia, the patient was informed about the complication, and the hospital management as well as the State Institute for Drug Control, as the local regulatory agency, were duly notified of possible device malfunction.

\section{Discussion}

From the anatomical point of view, the areas associated with highest risk for guide wire entrapment include, essentially, the soft tissue between the clavicle and the first rib, coracoclavicular ligament, territory of the confluence of subclavian vein, external and internal jugular vein, and the thoracic duct (referred to as the venous angle). The diameter of the venous lumen subsequently widens towards the right atrium and thus the risk of entrapment is lower in that area.

As already stated in the Introduction section, complications associated with guide wire introduction/removal are usually dealt with after prolonged periods of time, hours to days. Initially, an X-ray image is obtained to choose between the technique of fluoroscopy-guided intervention with local anesthesia, or surgery with general anesthesia.

Our case differs in that central venous catheter placement was undertaken at the beginning of the procedure after general anesthesia had already been induced. Also, given the nature of the intended procedure, a skilled team of thoracic surgeons was available. Last, but not least, the bend of the guide wire was clearly palpable in the subclavian triangle.

Therefore, we were confident the guide wire was entrapped extravasally between the clavicle and the first rib. Knowing the reason of the incident, we decided not to try to visualize the guide wire any further and to immediately manage the problem.

Our decision allowed us to successfully deal with the complication within a mere 30 minutes of the incident. The question remains, whether the possible device malfunction was the primary cause of the entrapment. We believe that although the anatomical circumstances might have been challenging in this particular patient, the guide wire should nevertheless withstand forces needed for its placement. So, we suppose that fraying of the guide wire was either the primary cause or had at least substantially contributed to the whole incident.

\section{Conclusion}

The physician introducing a central venous catheter has to be familiar with all types of complications, not only the common (as pneumothorax) but, also, the rare ones (as was our case). Managing these complications requires skill, ingenuity and, sometimes, interdisciplinary cooperation, either with a radiologist or a surgeon. The faster the complication is resolved, the better for the patient and, also, for the workflow of the health care facility. Our decision to proceed with immediate surgical removal of the guide wire proved a right one, and, to the best of our knowledge, such a strategy has not been described in the relevant literature to date. 
We feel other physicians could possibly opt for the same procedure when faced with situations similar to those described in our case report.

\section{References}

Katiyar, S., Jain, R. (2010) Entrapped central venous catheter guide wire. Indian J. Anaesth. 54, 354-355.

Khan, K. Z., Graham, D., Ermenyi, A., Pillay, W. R. (2007) Case report: Managing a knotted Seldinger wire in the subclavian vein during central venous cannulation. Can. J. Anaesth. 54, 375-379.

Leigh, T. R., Baker, L. R., Cattell, W. R. (1987) Internal knotting of a guidewire: A complication of subclavian vein cannulation. Nephron 46, 210.

Önal, Ö., Çelebioğlu, B., Kanbak, M., Demircin, M., Aypar, Ü. (2013) An unusual complication during femoral venous catheterisation: Separation of spiral wire surrounding guide wire and its late diagnosis in a child. OA Case Reports 2, 134.

Pal, R., Laha, B., Nandy, S., Biswas, R. (2014) Coiling of guide wire in the internal jugular vein during central venous catheter insertion: a rare complication. Indian J. Anaesth. 58, 786-788.

Polos, P. G., Sahn, S. A. (1991) Complication of central venous catheter insertion: Fragmentation of a guidewire with pulmonary artery embolism. Crit. Care Med. 19, 438-440.

Schummer, W. (2002) Loss of the guide wire: mishap or blunder? Br. J. Anaesth. 88, 144-146. 